\title{
Pandemic palliative care: beyond ventilators and saving lives
}

\author{
Amit Arya MD, Sandy Buchman MD, Bruno Gagnon MD MSc, James Downar MDCM MHSc (Bioethics)
}

Cite as: CMAJ 2020 April 14;192:E400-4. doi: 10.1503/cmaj.200465; early-released March 31, 2020

CMAJ Podcasts: interview in English at https://soundcloud.com/cmajpodcasts/200465-ana and in French at https://soundcloud.com/ cmajpodcasts/200465-ana-fre

$\mathbf{T}$ he current novel coronavirus, severe acute respiratory syndrome coronavirus 2 (SARS-CoV-2) pandemic will likely strain Canada's health care system beyond capacity, and many people are expected to die as a result. In this article, we review the challenges involved in providing palliative care in a pandemic.

In Italy, hospitals were forced to triage patients at an early stage, with many older patients with comorbidities being denied access to critical care resources. ${ }^{1}$ Indeed, even in a well-resourced country, the number of people denied life-sustaining treatments in a pandemic could exceed the number who receive them. ${ }^{2}$ Some might view this as a failure of the system, but this would be unfair. No sustainable system of health care can hope to accommodate such a surge in demand. But those who are denied access to critical care have a right to expect high-quality palliative care in place of a ventilator. Failing to deliver palliative care in this context would compound the tragedy of the pandemic and would arguably be a more substantial failure of the health care system.

\section{What are the challenges to providing palliative care during a viral pandemic?}

Palliative care focuses on 3 domains: management of patient symptoms; discussion of patient wishes, expectations and values via advance care planning and articulation of goals of care; and supporting families of those with life-limiting illness. ${ }^{3}$ We expect the current SARS-CoV-2 pandemic to produce a surge of patients who present with acute respiratory failure due to pneumonia, including symptoms such as fever, dyspnea, respiratory congestion, pain, nausea and delirium. The risk of death is higher for older adults, particularly those with frailty and comorbidities. Many people already have advance care plans that stipulate that comfort measures are to be used if they become seriously ill. Other patients who are intubated and receiving mechanical ventilation but are not improving clinically will be extubated. A third group of patients may be denied ventilation because of resource scarcity. Thus, in a viral pandemic, we expect the need for palliative care to increase substantially. Moreover, palliative care services will need to be available across many different care settings, including intensive care units (ICUs), hospital wards, emergency departments and long-term care facilities, where some of the patients

\section{KEY POINTS}

- The severe acute respiratory syndrome coronavirus 2 (SARSCoV-2) pandemic will likely strain our health care system beyond capacity, and palliative care services will be needed across many different care settings, including intensive care units, hospital wards, emergency departments and long-term care.

- Shared decision-making between clinicians and patients is a core process in planning for the end of life; however, in a pandemic, patient autonomy to choose life-prolonging measures or location of death could be severely restricted as a result of public health directives and resource availability, and some patients may necessarily be isolated at end of life.

- Previous mass casualty events have taught us much about how best to triage patients requiring care, and some of this work can be adapted to palliative care; but little has been written on how to manage those who are not offered life-sustaining measures.

- We advise acting now to stockpile medications and supplies used in palliative care, train staff to meet palliative care needs, optimize our space, refine our systems, alleviate the effects of separation, have critical conversations and focus on marginalized populations to ensure that all patients are cared for equitably.

- The SARS-CoV-2 pandemic has been tragic for many people worldwide. Failing to provide Canadians with effective palliative care would compound that tragedy.

at highest risk reside. The latter, however, are chronically understaffed and may be undersupplied with the medications and palliative care delivery systems needed to control severe symptoms.

Shared decision-making between clinicians and patients is a core process in planning for the end of life; however, in a pandemic scenario, patient autonomy to choose life-prolonging measures or location of death could be severely restricted as a result of public health directives and resource availability. Patients may not be offered mechanical ventilation even if they desire it. Patients with coronavirus disease 2019 (COVID-19) may need to be confined to a space (e.g., a ward for patients with the disease) that is not of their choosing. Furthermore, as epidemiological information about SARS-CoV-2 is still evolving, prognostication could be quite difficult, leading to uncertain outcomes for patients with COVID-19associated pneumonia and respiratory failure. These situations will 
Box 1: A palliative care pandemic plan for management of coronavirus disease 2019 (COVID-19; updated from Downar and Seccareccia) ${ }^{2}$

\section{Stuff}

- Stockpile comfort medications (morphine, haloperidol, midazolam and scopolamine) or symptom management kits, especially in longterm care and community settings

- Suspend regulations that limit the availability and prescription of injectable morphine and hydromorphone

- Stockpile equipment to deliver medications, including subcutaneous cannulae and delivery equipment (e.g., pumps or syringe drivers)

- Stockpile personal protective equipment for palliative care providers in long-term care and community settings

Staff

- Identify and mobilize all clinicians with palliative care experience

- Provide brief education for front-line providers on symptom management for acute respiratory illness, emphasizing the safety of symptom-targeted opioids as an early option

- Engage allied health to provide emotional support to patients, and grief and bereavement support to family members

\section{Space}

- Optimize the use of beds in hospice and palliative care units, particularly for patients who do not have COVID-19, via direct admission from the emergency department or community

- Identify separate wards and nonclinical areas in acute settings that might be appropriate for those expected to die - essentially palliative care units for patients with COVID-19

\section{Systems}

- Adopt a triage system to determine which patients require specialist palliative care consultation and which patients can be seen virtually

- Maximize the use of telemedicine, both for efficiency and reducing infection

- Develop standardized order sets for acute, long-term care and community settings

- Form palliative care provider "groups" that can provide mutual support and coverage if a provider becomes sick or is overwhelmed

\section{Sedation}

- Be prepared to use palliative sedation for symptoms that are refractory to common comfort medications

\section{Separation}

- Enable video calling to connect patients with family members who are separated because of travel and visitor restrictions

\section{Communication}

- People with frailty or comorbid illness should update their advance care plans and indicate if they wish to avoid transfers to hospital or critical care in the event of serious illness

- Before the expected surge in patients, providers should review treatment plans when patients with advanced cancer, end-stage organ failure, frailty or dementia are requesting life support or cardiopulmonary resuscitation. These patients are unlikely to survive and recover after an admission to an intensive care unit.

\section{Equity}

- Palliative care providers should pay greater attention to patients who are marginalized. When the health care system is strained, systemic inequity will worsen.

- Protocols for critical care triage may be implemented. Patients who are denied critical care should be the top priority for palliative care. All patients must be cared for. likely cause considerable distress among patients, families and health care teams.

Patients and families have emotional, psychological, social and spiritual needs, which often are addressed by an interprofessional palliative care team approach involving a physician, nurse, social worker and spiritual care worker. In a pandemic, patients dying of viral pneumonia caused by SARS-CoV-2 may face isolation because of visitor and travel restrictions. Furthermore, overstretched health care teams may be unable to spend time at the bedside or to physically examine patients regularly, which could negatively affect how patients perceive their care. ${ }^{4,5}$

\section{What can we learn from previous experiences with providing palliative care during pandemics and other humanitarian emergencies?}

Previous mass casualty events, such as acts of terrorism, natural disasters or epidemics, have so overwhelmed health care systems that they could no longer provide equal access to care. Generally, these situations fall into 1 of 2 categories: "big bang" events such as airplane or train crashes, and "rising tide" incidents such as viral epidemics. ${ }^{6}$ The main goal of a coordinated response from public health and disaster-planning personnel is (appropriately) saving the maximum number of lives possible. Yet, despite a large body of literature and experience with triage, little has been written on how to manage those who are not offered life-sustaining measures. Indeed, one source of anxiety to both health care providers and members of the public is the concern that triage processes might lead some patients to receive no care at all or perhaps to have their lives ended intentionally without their consent. ${ }^{2}$ This would compound the tragedy of triage in a pandemic - patients are first denied life-sustaining therapy and then they are not provided appropriate symptom control. They die at the wrong time, in the wrong place, and they may get the wrong care. Patients should always have access to highquality palliative care as a basic human right, ${ }^{7}$ but, in a triage situation, our obligation to provide palliative care for those denied lifesustaining measures is increased. Past experiences with viral epidemics, such as Ebola virus, severe acute respiratory syndrome (SARS) and HIV, have emphasized the integration of palliative care as an essential part of any health care intervention. ${ }^{8-10}$

\section{How could palliative care be provided during a pandemic?}

In 2008, the US Task Force on Mass Casualty Critical Care publisheda framework for mass casualty events that comprised "stuff," "staff," "space" and "systems," ${ }^{11}$ which was adapted previously to the palliative care context. ${ }^{2}$ As experienced palliative care providers, we consider it important to add 4 additional elements: "sedation," "separation," "communication" and "equity" (Box 1).

Stuff refers to medications needed to provide comfort and the means to administer them to a large number of patients. This may be problematic during the SARS-CoV-2 pandemic because regulations in many countries preclude the creation of easily accessible stockpiles of opioids. In addition, long-term care facilities may not have sufficient drugs on hand to treat more than a few patients for a long period, and 
Table 1: Suggested contents of a symptom management kit and recommended starting doses for patients with severe coronavirus disease $2019^{*}$

\begin{tabular}{|c|c|c|c|}
\hline Symptom & Treatment & Recommended starting order & Quantity for kit \\
\hline Pain or dyspnea & Hydromorphone $2 \mathrm{mg} / \mathrm{mL}$ & $0.5-1.0 \mathrm{mg}$ subct q30min p.r.n. $\dagger$ & 10 vials \\
\hline Nausea or delirium & Haloperidol $5 \mathrm{mg} / \mathrm{mL}$ & $1 \mathrm{mg}$ subcut q2h p.r.n. $\neq$ & 10 vials \\
\hline Sedation & Midazolam $5 \mathrm{mg} / \mathrm{mL}$ & $1-2$ mg subcut q15min p.r.n.§ & 10 vials \\
\hline Secretions & Scopolamine $0.4 \mathrm{mg} / \mathrm{mL}$ & $0.4 \mathrm{mg}$ subcut q4h p.r.n. & 10 vials \\
\hline Fever & Acetaminophen $650 \mathrm{mg}$ suppositories & Administer q6h PR p.r.n. & 10 vials \\
\hline Urinary retention & Foley catheter 16 French & Insert catheter p.r.n. & 1 kit \\
\hline Dry mouth & Mouth swabs & Mouth care q.i.d. and p.r.n. & 10 swabs \\
\hline
\end{tabular}

these drugs may not be available quickly at the bedside. Preparing and distributing sufficient numbers of "palliative medication kits" could help address this issue in any setting with substantial numbers of patients who might not survive. ${ }^{12,13}$ Ideally, these kits would include opioids to alleviate pain or dyspnea, haloperidol for nausea or agitated delirium, scopolamine for respiratory secretions, acetaminophen for fever and chills, and midazolam for sedation (Table 1). Kits should also include subcutaneous cannulae to deliver these drugs. Infusion systems (e.g., pumps or spring-loaded syringes) should also be available for patients who need continuous medications. We suggest stockpiling sufficient numbers of kits in appropriate locations to ensure availability for a variety of clinicians including paramedics, staff of longterm care facilities and family physicians. Personal protective equipment (PPE) should also be stocked and available to palliative care providers, especially those who visit patients at home. If community providers are unable to provide treatment, their patients will present to acute care and exacerbate the crisis further.

In terms of staff, because palliative care specialist teams cannot provide direct care to all patients who are dying during a surge, regional pandemic planning should include engagement of all interprofessional health care teams who have training and experience in end-of-life care. Spiritual care staff and social work should be ready to manage common psychosocial needs such as grief and bereavement. To build further capacity, focused education on endof-life care for patients with COVID-19 should be provided to frontline health care providers such as primary care physicians, nurse practitioners, paramedics, emergency department staff and nurses in long-term care facilities. This education should focus on the use and titration of opioids for dyspnea. Several studies highlighted the safety and efficacy of opioids for treating dyspnea in advanced lung disease, ${ }^{14,15}$ improving respiratory mechanics in chronic lung disease,$^{15}$ and reducing dyspnea and tachypnea without causing a rise in the level of carbon dioxide. ${ }^{16,17}$ All front-line providers should feel comfortable using symptom-targeted opioids early for dyspnea without waiting for respiratory failure to develop. Breathing is essential for life, but respiratory distress is not.

A 2006 report about hospice use in Taiwan during the SARS outbreak found that palliative care space had the potential to be underused. ${ }^{18}$ During an epidemic, people try to avoid health care facilities, and normal referral and transportation systems are disrupted. Paradoxically, even though more people are dying, they are likely to die in circumstances that prevent them from accessing hospice beds. Thus, new space may be needed to care for large numbers of patients who are dying of COVID-19-associated respiratory failure. This may be a specialized inpatient ward or a separate location adjacent to a hospital if transport to hospital is not feasible. Patients at home or living in a long-term care facility could be cared for on site, although they may need to be sequestered from others. A quiet and peaceful environment is recommended to support patient dignity in the last hours and days of life.

Box 2: Suggested triage tool for referral to specialist palliative care $^{19}$

All clinicians providing palliative care should address physical, social, financial and spiritual concerns

Clinicians who are not palliative care specialists (hospitalists, family physicians, internists, ICU physicians, nurse practitioners, nurses and paramedics) support the following:

- Identification and management of pain, dyspnea, agitated delirium and respiratory congestion

- Management of caregiver grief

- Discussions about prognosis, goals of treatment, suffering and resuscitation status

Palliative care specialist clinicians support the following:

- Patients with complex or refractory symptoms

- Patients who are denied access to critical care owing to a triage protocol, despite wanting aggressive care

- Management of complex depression, anxiety, grief and existential distress

- Requirement for palliative sedation therapy

- Pre-existing opioid use disorder

- Patients with young children

- Patients belonging to marginalized populations, including the homeless, incarcerated persons and Indigenous Peoples, who are at risk of being underserved by the health care system 
New systems are needed, including a triage system to allocate clinicians who could provide both primary- and specialist-level palliative care (Box 2). Symptom management for most patients with pneumonia is straightforward; a standardized order set (Table 1) and titration parameters (https://palliativecare.med.ubc.ca/coronavirus/) might reduce the need for referral to a specialist. Virtual visits can optimize efficiency and reduce risk of infection during a pandemic, and providers who are required to self-isolate or quarantine can continue to provide care. Palliative care providers should also anticipate that they might become sick or be asked to cover for sick or quarantined colleagues. As many palliative care providers work with little or no backup, this could have a dramatic effect on service provision. Providers should form (or join) larger groups of palliative care providers to help with cross-coverage and fluctuations in workload. This would also facilitate collegial wellness check-ins.

For symptoms of COVID-19 that are refractory to common comfort medications, palliative sedation is appropriate. Palliative sedation involves the use of sedatives at end of life to reduce a patient's level of consciousness so that they are not suffering. Protocols are accessible on the Internet, ${ }^{20}$ and, if available, palliative care consultants should support providers who are not experienced in providing this procedure. In our opinion, palliative sedation is preferable to medical assistance in dying (MAiD) for patients with severe respiratory failure caused by SARS-CoV-2, given the 10-day reflection period, number of witnesses and assessors required, and the current requirement for full capacity to determine eligibility for MAiD. Attempting to honour an urgent MAiD request is likely to prolong suffering in those who are imminently dying.

Box 3: Suggested language for physicians providing support to a patient or family member who is denied intensive care because of resource scarcity

Normally, when somebody develops critical illness, the medical team would offer them intensive care (a combination of medications and machines to support their vital organs), provided that the medical team felt that they had a reasonable chance of survival. However, because of the COVID-19 outbreak, we are currently unable to offer intensive care to everyone who is critically ill. As a result, our hospital is working under triage guidelines, which means that we are offering intensive care only to those who are most likely to be able to survive and recover from their critical illness. You probably have heard about this in the news - all hospitals in the region are working under these guidelines.

I regret to inform you that we are unable to offer you intensive care treatments at this time, as a result of the triage guidelines. Because of your medical condition, the likelihood that you would survive even with intensive care is considered to be too low for us to offer intensive care. The team has made this decision based on the following information:

I am deeply sorry about this situation. This is not the way we ordinarily make these decisions, and I can only imagine how you must feel right now. I want you to know that even though we cannot offer intensive care, we will do everything else that could conceivably give you a chance of recovering, including:

And I promise you that, no matter what, we will also use medication to treat any discomfort, such as pain or shortness of breath. We know that when we treat discomfort appropriately, this is not harmful and may actually help improve your condition.
Isolation measures, limitations on visitors and travel restrictions could lead to an increased sense of separation in patients who are nearing end of life and their families, which occurred during the SARS epidemic in 2003. ${ }^{9}$ Since then, technological advances have made video calling commonplace, which should help alleviate the sense of isolation for many. We suggest that health care facilities offer smartphones, tablets or laptops to patients and provide free Internet connections during the pandemic. However, some patients will not be able to use video calling because of their condition, so social workers and spiritual health professionals should prioritize these individuals for support. In addition, should PPE be available, we suggest that family members be allowed to visit. We should also recognize that the effect of separation will not end when the patient dies and anticipate the need to support family members through their disrupted grief and bereavement.

Patients who are frail, older or have multiple comorbidities are at the highest risk of death from SARS-CoV-2. Many of these patients will not ask for aggressive life-prolonging measures such as mechanical ventilation. Instead, they will prioritize honest communication, having time to say goodbye to loved ones and avoiding being a burden on their family. ${ }^{21}$ In a situation of resource scarcity, patients and family members may be surprised to learn that they will not be offered life-sustaining measures. This will be a challenging scenario for many physicians, and a sensitive, supportive approach may help reduce the distress of the patient and family (Box 3 ). However, if they are informed that their chances of surviving critical illness are poor, many will choose not to experience the burdens of intensive care. ${ }^{22}$ Therefore, when assessing patients who are critically ill with respiratory failure caused by SARS-CoV-2, it will be important for all physicians to ensure that any proposed treatment is clinically indicated

Box 4: Suggested language for discussing a treatment plan with someone who is unlikely to survive a critical illness, but whose current care plan would include lifesustaining therapies if indicated

You (your loved one) is currently suffering from ___. We have given you treatments, including ___ but it seems as though your body is not responding well to them. If this continues, we would need to consider the use of life-sustaining treatments to support your body.

I am very concerned about this scenario; although it is very easy to start life-sustaining treatments, there are many scenarios where we strongly prefer not to because the chances of recovery are poor. That is usually when someone has chronic or incurable medical conditions, or their body has become weaker than it used to be. The other concern is that these treatments can cause a lot of discomfort. Of course, many people are willing to experience discomfort if there is a reasonable chance of a good recovery. But if the treatments cause discomfort and the chances of recovery are poor, we are very hesitant to offer those treatments.

I would like to propose an alternative plan. I would like to suggest that we continue doing the things that we are currently doing, including , in the hope that you might still respond and recover. We do not want to take away that opportunity. But if your body does not respond and you get worse, I would suggest that we do not start life-sustaining treatments. Instead, if you get worse, I would suggest that we focus on keeping you comfortable, understanding that any further escalation of care would probably do more harm than good. What do you think about that? 
and in agreement with patient wishes and expectations (Box 4). The first fatal case of COVID-19 in Quebec was an elderly woman who did not wish to have aggressive interventions. Her decision was made after conversations with the treating team while she was provided with optimal care. ${ }^{23}$

Equity is an important principle to apply when triaging during a pandemic. At the best of times, people who are structurally vulnerable or who suffer with severe mental illness face substantial challenges in accessing health care in Canada. Patients in need of palliative care and simultaneously living with "deficits in the social determinants of health" have particular difficulty in accessing that care. ${ }^{24}$ In a pandemic, as our health care system becomes more stressed, inequities will become more pronounced. People facing poverty, discrimination, language barriers and historical trauma are least able to self-advocate and are at a greater disadvantage when facing difficult decisions about resource allocation. These patients are aware of this; studies have shown that they fear not getting access to life-sustaining treatments ${ }^{25}$ and may be judged as less deserving of care. ${ }^{26}$ Palliative care thus becomes the compassionate option to counterbalance this inequity.

\section{Conclusion}

Palliative care should be an essential part of any response to a humanitarian crisis, including the current SARS-CoV-2 pandemic. A multipronged approach that is focussed on stuff, staff, space, systems, sedation, separation, communication and equity can guide planning and ensure that the palliative care needs of patients and their family members are met. Any triage system that does not integrate palliative care principles is unethical. Patients who are not expected to survive should not be abandoned but must receive palliative care as a human right.

\section{References}

1. Rosenbaum L. Facing Covid-19 in Italy - ethics, logistics, and therapeutics on the epidemic's front line. [Epub ahead of print]. N Engl J Med 2020 Mar. 18. doi: 10.1056/NEJMp2005492.

2. Downar J, Seccareccia D. Palliating a pandemic: "all patients must be cared for". J Pain Symptom Manage 2010;39:291-5.

3. Hawley P. Barriers to access to palliative care. Palliat Care $2017 ; 10$ : 1178224216688887. doi: 10.1177/1178224216688887.

4. Strasser F, Palmer JL, Willey J, et al. Impact of physician sitting versus standing during inpatient oncology consultations: patients' preference and perception of compassion and duration. A randomized controlled trial. J Pain Symptom Manage 2005;29:489-97.
5. Kadakia KC, Hui D, Chisholm GB, et al. Cancer patients' perceptions regarding the value of the physical examination: a survey study. Cancer 2014;120:2215-21.

6. Matzo M, Wilkinson A, Lynn J, et al. Palliative care considerations in mass casualty events with scarce resources. Biosecur Bioterror 2009;7:199-210.

7. Henteleff $P$, Shariff MJ, Macpherson DL. Palliative care. An enforceable Canadian human right? McGill J Law Health 2011;5:106-60.

8. Escobio F, Nouvet E. Ebola virus disease and palliative care in humanitarian crises. Lancet 2019;393:1100. doi: https://doi.org/10.1016/S0140-6736(19)30295-8.

9. Leong IY, Lee $\mathrm{AO}, \mathrm{Ng} \mathrm{TW}$, et al. The challenge of providing holistic care in a viral epidemic: opportunities for palliative care. Palliat Med 2004;18:12-8.

10. Merlins JS, Tucker RO, Saag MS, et al. The role of palliative care in the current HIV treatment era in developed countries. Top Antivir Med 2013;21:20-6.

11. Christian M, Devereaux AV, Dichter JR, et al. Definitive care for the critically ill during a disaster: current capabilities and limitations: from a Task Force for Mass Critical Care summit meeting, January 26-27, 2007, Chicago, IL. Chest 2008;133(Suppl):8S-17S

12. Bullen T, Rosenberg JP, Smith B, et al. The use of emergency medication kits in community palliative care: an exploratory survey of views of current practice in australian home-based palliative care services. Am J Hosp Palliat Care 2015;32:581-7.

13. Wowchuk SM, Wilson EA, Embleton L, et al. The palliative medication kit: an effective way of extending care in the home for patients nearing death. J Palliat Med 2009;12:797-803.

14. Mahler DA, O'Donnell DE. Recent advances in dyspnea. Chest 2015;147:232-41.

15. Currow DC, McDonald C, Oaten S, et al. Once-daily opioids for chronic dyspnea: a dose increment and pharmacovigilance study. J Pain Symptom Manage 2011;42:388-99.

16. Abdallah SJ, Wilkinson-Maitland C, Saad N, et al. Effect of morphine on breathlessness and exercise endurance in advanced COPD: a randomised crossover trial. Eur Respir J 2017;50:1701235.

17. Clemens KE, Klaschik E. Symptomatic therapy of dyspnea with strong opioids and its effect on ventilation in palliative care patients. J Pain Symptom Manage 2007;33:473-81.

18. Chen TJ, Lin MH, Chou LF, et al. Hospice utilization during the SARS outbreak in Taiwan. BMC Health Serv Res 2006;6:94.

19. von Gunten CF. Secondary and tertiary palliative care in US hospitals. JAMA 2002;287:875-881.

20. Palliative sedation at the end of life. Montréal: Collège des Médecins du Quebec; 2016. Available: www.cmq.org/publications-pdf/p-1-2016-08-29-en-sedation -palliative-fin-de-vie.pdf (accessed 2020 Mar. 22).

21. Heyland DK, Dodek P, Rocker G, et al. What matters most in end-of-life care: perceptions of seriously ill patients and their family members. CMAJ 2006; 174:627-33.

22. Murphy DJ, Burrows D, Santilli S, et al. The influence of the probability of survival on patients' preferences regarding cardiopulmonary resuscitation. N Engl J Med 1994;330:545-9.

23. Archambault $\mathrm{H}$. The deceased patient refused to give up [in French]. Le Journal de Montréal 2020 Mar. 18. Available: www.journaldemontreal.com/2020/03/18/ la-patiente-decedee-a-refuse-lacharnement (accessed 2020 Mar. 30).

24. Reimer-Kirkham S, Stajduhar K, Pauly B, et al. Death is a social justice issue: perspectives on equity-informed palliative care. ANS Adv Nurs Sci 2016;39:293-307.

25. Ko E, Kwak J, Nelson-Becker H. What constitutes a good and bad death?: perspectives of homeless older adults. Death Stud 2015;39:422-32.

26. Browne AJ, Varcoe CM, Wong ST, et al. Closing the health equity gap: evidence based strategies for primary health care organizations. Int J Equity Health 2012;11:59.
Competing interests: Sandy Buchman is the president and a member of the Board of Directors of the Canadian Medical Association. He has received speaker fees from the University of Toronto and Cancer Care Ontario, and nonfinancial support from the Israel National Institute for Health Policy Research. James Downar has received consultant fees from Joule and honoraria from Boehringer Ingelheim (Canada). Amit Arya has received honoraria from Pallium Canada for peer review activities for LEAP course materials. No other competing interests were declared.

This article was solicited and has not been peer reviewed.

Affiliations: Division of Palliative Care (Arya, Buchman), Department of Family Medicine, McMaster University, Hamilton, Ont.; Division of Palliative Care (Arya, Buchman), Department of Family and Community Medicine, University of Toronto, Toronto, Ont.; Departments of Family
Medicine and Emergency Medicine (Gagnon), Cancer Research Centre, Université Laval, Québec, Que.; Division of Palliative Care (Downar), Department of Medicine, University of Ottawa; Department of Palliative Care (Downar), Bruyère Continuing Care Ottawa, Ont.

Contributors: All of the authors conceived the work, drafted the original manuscript, reviewed it critically for important intellectual content, gave final approval of the version to be published and agreed to be accountable for all aspects of the work.

Funding: This work was unfunded.

Acknowledgments: The authors thank Dr. Cecile Bensimon for reviewing and providing feedback on this manuscript, and Dr. Jill Rice for the suggested order set in Table 1.

Correspondence to: James Downar, jdownar@toh.ca 